\title{
Fast Functional Modelling of Diode-Bridge Rectifier Using Dynamic Phasors
}

\author{
T. Yang, S. V. Bozhko, G. M. Asher \\ The University of Nottingham, Nottingham NG7 2RD, UK
}

This paper is a postprint of a paper submitted to and accepted for publication in IET Power Electronics and is subject to Institution of Engineering and Technology Copyright. The copy of record is available at IET Digital Library

\author{
Corresponding Author: $\quad$ Dr Tao Yang \\ Power Electronics, Machine and Control Group \\ Faculty of Engineering \\ The University of Nottingham \\ Nottingham NG7 2RD \\ United Kingdom \\ Phone: $\quad$ +44(0)115748 4739 \\ Email_Tao.Yang@nottingham.ac.uk
}

Abstract - In this paper, a functional model for diode-bridge rectifiers is developed based on the dynamic phasor concept. The developed model is suitable for accelerated simulation studies of the electric power systems under normal, unbalanced and line faulty conditions. The high accuracy and efficiency of the developed model have been demonstrated by comparison against threephase time-domain model and against the model employing synchronous space-vector representations. The experimental verification of the developed model is also reported. In addition, an error analysis shows that the error of the developed model is less than $10 \%$ at the most severe unbalanced conditions. The prime purpose of the model is for the simulation studies of moreelectric aircraft power architectures at a system level; however it can be directly applied for simulation study of any other electrical power system interfacing with uncontrolled diode bridge rectifiers.

Index Terms - AC-DC power conversion, Aircraft Power Systems, Converters, Dynamics, Modelling, Rectifiers, Vectors 



\section{INTRODUCTION}

There has been seen a significant penetration of power electronics into electric power systems (EPS) in recent years. For example, terrestrial EPS's particularly at distribution level, promise a multiplicity of power electronic converters to handle renewable sources, energy storage or EPS conditioning. A similar scenario pertains for isolated and mobile EPS, such as more-electric aircraft (MEA) and more-electric ship. In the MEA the electric power conversion is required to manage power distribution, landing gear, flight actuation and other functions [1].

The use of large numbers of power electronic devices brings significant modelling challenge at the EPS system level due to the system complexity and the wide variation in time constants. The challenge is to balance the simulation speed against the model accuracy and this is dependent on the modelling task. Four different modelling layers are defined according to the modelling bandwidths i.e. architectural models, functional models, behavioural models and component models [2], [3]. The architectural layer computes steady state power flow and is used for weight, cost and cabling studies. In the functional level, the system components are modelled to handle the main system dynamics up to $150 \mathrm{~Hz}$ and the error should be less than 5\% in respect of the behaviour model accuracy. The behavioural model uses lumped-parameter subsystem models and the modelling frequencies can be up to hundreds of $\mathrm{kHz}$. The component models cover high frequencies, electromagnetic field and electromagnetic compatibility (EMC) behaviour, and perhaps thermal and mechanical stressing. The bandwidth of component models can be up to in $\mathrm{MHz}$ region if required.

Targeting for acceptable simulation times for system-level EPS modelling, a number of approaches have been investigated. Average state-space models [4] are a standard technique for considering only the fundamental wave converter behaviour. Average modelling of ac distribution systems involves transforming the three-phase ac signals to a synchronous rotating $d q$ frame, henceforth termed the $d q 0$ model. This method has been used in modelling MEA electric power systems and is proved to be an effective technique [3]. A model with three-phase ac variables is henceforth called an $a b c$ model.

One of the disadvantages of the $d q 0$ approach is that under faulty and unbalanced conditions double-frequency components appear and the simulation time steps must be drastically reduced to maintain accuracy. An alternative approach that can address this problem is dynamic phasors (DPs) [5, 6]. The DP method has been applied to the modelling of terrestrial EPS systems including imbalanced regimes [7, 8]. Comparative study of a simple EPS with line faults carried out in $a b c, d q 0$ and DP domain is given in [9] where the efficiency of the DP approach is demonstrated. The DP method has also been applied for modelling of electrical machines [10,11] and flexible ac transmission systems [12], including active filters [13] and static synchronous compensator (STATCOM) [14]. In [15] and [16], a DP model for thyristor-based high-voltage direct current (HVDC) transmission systems is reported, although only balanced conditions are considered. 
Different models for uncontrolled diode-bridge rectifiers (DBs) have been developed in recent publications. A comprehensive analytical model is developed in [17] and [18], where different operation modes of DBs have been thoroughly studied. Another mathematical model for analysing DBs based on switching functions is introduced in [19]. An average model for DB is proposed in [20]. However, all these models are only for steady-state applications and the power supply is assumed to be balanced. This paper aims to develop a DP model for DBs. This model can be used for both steady-state and transient studies, under balanced and unbalanced conditions. At the time of this study development, no report on DB modelling in DP domain has been published. The most similar to DBs modelled using DP approach is a thyristor-based HVDC converter [16]. In addition, the model reported in [16] is suitable only for balanced operations. The DB functional model presented in this paper is based on the DB relations between ac and dc terminal variables. The negative sequence under unbalanced or fault conditions is treated as a disturbance in the model. Both the $2^{\text {nd }}$ and $6^{\text {th }}$ harmonic on the dc-link voltage are conveniently included in the reported DP model. This enables to cover the ac imbalanced voltage and dc ripple voltage for both continuous and discontinuous operation.

The main results of the paper are summarised as follows:

- The DP approach has been successfully applied to develop model an uncontrolled DB rectifier applicable for accelerated simulation studies of complex EPS, both balanced and unbalanced, and is consistent with the functional modelling layer specifications in [3]. The model is independent on other EPS devices and may be used as a library element interfacing within an extended three-phase EPS models

- The model is verified experimentally under both balanced and unbalanced operation

- The computational effectiveness of the developed model is proved through comparison against time-domain $a b c$ switching and functional non-switching $d q 0$ models and a significant computational acceleration is demonstrated.

\section{DYNAMIC PHASORS}

This Section, in order to keep the paper self-containment and to assist readability, shortly revises the basic concepts that are employed for the development of the diode bridge model in DP domain. For the readers who are not familiar with the concept, it is recommended to refer to the basic DP theory [5] since it will be intensively used throughout the rest of the paper.

The DP concept assumes that a time-domain nearly-periodic waveform $x(\tau)$ can be represented on the interval $\tau \in(t$ $\mathrm{T}, t]$ by a following Fourier series: 


$$
x(\tau)=\sum_{k=-\infty}^{\infty} X_{k}(t) e^{j k \omega_{s} \tau}, \quad \tau \in(t-T, t]
$$

where $\omega_{s}=2 \pi / T$ and $T$ the fundamental period of the waveform. $X_{k}(t)$ is the $k^{\text {th }}$ Fourier coefficient in complex form referred to as a "dynamic phasor" and determined as follows:

$$
X_{k}(t)=\frac{1}{T} \int_{t-T}^{t} x(\tau) e^{-j k \omega_{s} \tau} d \tau=\langle x\rangle_{k}
$$

where $k$ is the DP index and angular brackets \langle\rangle are used to denote DP-domain variables. In contrast to the traditional Fourier Transformation (FT), these Fourier coefficients are time-varying as the integration interval (window) slides through time. As it follows, the DP represents the variation in specific frequency component over time. The required accuracy of the timedomain variable approximation can be achieved by appropriate selection of a DP set $K$ for a particular modelling task. For example, for dc-like variables and signals the index set only includes the component $k=0$, and for purely sinusoidal ones $k=1$.

A key factor in developing dynamic models based on DP is the relation between the derivatives of the variable $x(\tau)$ and the derivatives of $k^{\text {th }}$ Fourier coefficients [5]:

$$
\left\langle\frac{d x}{d t}\right\rangle_{k}=\frac{d X_{k}(t)}{d t}+j k \omega_{s} X_{k}(t)
$$

This can be verified using (1), (2), and may be used in evaluating the $k^{\text {th }}$ phasor of time-domain model. Another important property of DP is that the $k^{\text {th }}$ phasor of a product of two time-domain variables can be obtained via the convolution of corresponding DPs:

$$
\langle x y\rangle_{k}=\sum_{i}\langle x\rangle_{k-i}\langle y\rangle_{i}
$$

The properties (3) and (4) play a key role when transforming the time-domain models into DP domain. Algebraic manipulations in this paper will also exploit the following property of real functions $x(\tau)$ :

$$
X_{-k}(t)=X_{k}^{*}(t)
$$

where the notation ${ }^{*}$ denotes a complex conjugate.

\section{UNCONTROLLED DIODE-BRIDGE RECTIFIER}

A key objective of this paper is development of a computationally efficient model that can represent the DB behaviour in both balanced and unbalanced supply conditions including line faults. The functional level modelling does not 
require consideration of switching behaviour [3]. Hence, our study is based on the non-switching model that is reviewed in this Section.

A three-phase diode bridge rectifier is well-documented ac/dc converter and it is shown in Fig.1. In general case, it is supplemented by an output dc filter $L_{d c} C$ and a front-end inductance $L_{s}$ representing feeding cables, ac chokes etc. The dc load is shown as an equivalent resistance $R_{L}$. This converter employs uncontrolled diodes; hence the switching instants are determined by the circuit condition exclusively. The time-domain non-switching DB model under balanced conditions is well developed and documented in many publications, for example [17, 21].

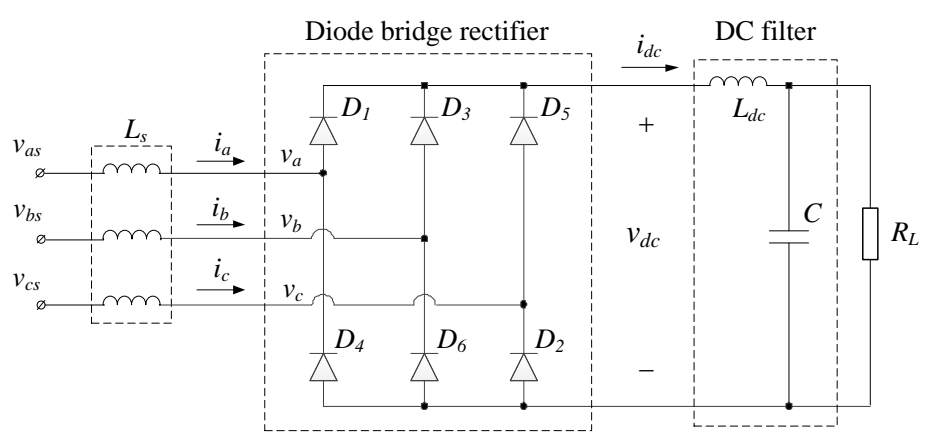

Fig. 1 Three-phase diode bridge rectifier

Under the symmetrical balanced supply the rectifier terminal voltages can be represented as

$$
\mathbf{V}_{a b c}=\left[\begin{array}{c}
v_{a} \\
v_{b} \\
v_{c}
\end{array}\right]=v_{m}\left[\begin{array}{c}
\cos (\omega t+\varphi) \\
\cos (\omega t+\varphi-2 \pi / 3) \\
\cos (\omega t+\varphi+2 \pi / 3)
\end{array}\right]
$$

where $v_{m}$ is the voltage magnitude, $\omega$ is the supply electrical frequency and $\varphi$ is the initial phase angle. The fundamental of diodes switching function then is given as follows [22]:

$$
\mathbf{S}_{a b c}=\left[\begin{array}{c}
S_{a} \\
S_{b} \\
S_{c}
\end{array}\right]=\frac{2 \sqrt{3}}{\pi}\left[\begin{array}{c}
\cos (\omega t+\varphi) \\
\cos (\omega t+\varphi-2 \pi / 3) \\
\cos (\omega t+\varphi+2 \pi / 3)
\end{array}\right]
$$

The switching function (7) defines the input-output relations for the diode bridge:

$$
\begin{aligned}
& v_{d c}=\mathbf{S}_{a b c}^{T} \mathbf{V}_{a b c}=\frac{3 \sqrt{3}}{\pi} v_{m} \\
& \mathbf{I}_{a b c}=\mathbf{S}_{a b c} i_{d c}
\end{aligned}
$$

where $\boldsymbol{I}_{a b c}=\left[\begin{array}{lll}i_{a} & i_{b} & i_{c}\end{array}\right]^{T}$ - an input ac current in vector-matrix form, $v_{d c}$ and $i_{d c}-$ rectifier output dc voltage and current. As it follows from (9) and (7), the input current is in phase with the input voltage (6) and its magnitude is: 


$$
i_{m}=\frac{2 \sqrt{3}}{\pi} i_{d c}
$$

In a presence of front-end inductance $L_{s}$ the dc-link voltage (8) reduces due to the commutation effect [22]. Normally, this effect is taken into account by introducing in (8) an additional term corresponding to a voltage drop across the resistance of $r_{f}$ value:

$$
r_{f}=6 f L_{s}
$$

In the following paper sections we will also intensively use the voltage (6) expressed as a space vector:

$$
\vec{v}=2 / 3 \cdot\left(v_{a}+v_{b} e^{j 2 \pi / 3}+v_{c} e^{-j 2 \pi / 3}\right)
$$

It is convenient to analyse three-phase power electronic circuits in synchronously rotating frame[3], [4]. Define the $d q$ frame such that it rotates with the grid electrical frequency $\omega$. Any three-phase variable $\mathbf{f}_{a b c}$ can be expressed in the $d q$ frame such that:

$$
\mathbf{f}_{d q}=\mathbf{T f}_{a b c}
$$

where $\mathbf{T}$ is a transformation matrix:

$$
\mathbf{T}=\frac{2}{3}\left[\begin{array}{rrr}
\cos \omega t & \cos (\omega t-2 \pi / 3) & \cos (\omega t+2 \pi / 3) \\
-\sin \omega t & -\sin (\omega t-2 \pi / 3) & -\sin (\omega t+2 \pi / 3)
\end{array}\right]
$$

Combining (8)-(10), (12)- (14) yields the following:

$$
\begin{aligned}
& v_{d c}=\frac{3 \sqrt{3}}{\pi} \sqrt{v_{d}^{2}+v_{q}^{2}} \\
& i_{m}=\sqrt{i_{d}^{2}+i_{q}^{2}}=\frac{2 \sqrt{3}}{\pi} i_{d c}
\end{aligned}
$$

where $v_{d}$ and $v_{q}$ are $d$ and $q$ components of voltage vector (12). Since the input current fundamental is in phase with the input voltage, its $d$ and $q$ components are as follows:

$$
i_{d}=i_{m} \cos \varphi ; \quad i_{q}=i_{m} \sin \varphi
$$

Under the balanced supply, $\varphi$ is equal to the initial phase of the input voltage and can be derived from voltage $d$ - and $q$ components:

$$
\varphi=\tan ^{-1}\left(v_{q} / v_{d}\right)
$$

Equations (15)-(17) comprise the relations between the DB input voltage/current expressed through their $d q$ components and 
the output dc voltage/current. These equations will be employed for the development of DB model in the DPs domain.

\section{DyNAMic Phasor DB Model DEVElopment}

In this Section the non-switching DP-domain DB model is developed. We will start with establishing how to map the generally unbalanced supply voltage vector (12) expressed in terms of synchronous $d q$ frame from time domain into the frequency domain of DPs and how the corresponding DPs can be derived from time-domain values of individual phase voltages $v_{a}, v_{b}, v_{c}$ for both balanced and unbalanced conditions. Then the DB input-output relations (15) will be transformed into DPs and the DP-domain DB model will be assembled using the derived relations.

\section{A. Input voltages and current in dynamic phasors domain}

In general cases, the DB terminal voltage can be represented by:

$$
v_{i}=V_{i} \cos \left(\omega t+\varphi_{i}\right), \quad i=a, b, c
$$

where $V_{a}, V_{b}$ and $V_{c}$ are phase voltage magnitudes, and $\varphi_{a}, \varphi_{b}, \varphi_{c}$ - their phase angles. Applying the Euler formulae, each of these components can be rewritten as:

$$
v_{i}=\frac{V_{i} e^{j \varphi_{i}} e^{j \omega t}+V_{i} e^{-j \varphi_{i}} e^{-j \omega t}}{2}, i=a, b, c
$$

The DPs of phase voltages can be derived applying the definition (2) and selecting the DPs set as $k=1$ since (18) includes only the fundamental component:

$$
\left\langle v_{i}\right\rangle_{1}=\frac{1}{2} V_{i} e^{j \varphi_{i}}, \quad\left\langle v_{i}\right\rangle_{-1}=\left\langle v_{i}\right\rangle_{1}^{*}=\frac{1}{2} V_{i} e^{-j \varphi_{i}}, i=a, b, c
$$

Combining equations (12), (18), (19) and re-arranging the terms results in the following relation:

$$
\vec{v}=\frac{2}{3} e^{j \omega t}\left[\left\langle v_{a}\right\rangle_{1}+\left\langle v_{b}\right\rangle_{1} e^{j 2 \pi / 3}+\left\langle v_{c}\right\rangle_{1} e^{-j 2 \pi / 3}\right]+\frac{2}{3} e^{-j \omega t}\left[\left\langle v_{a}\right\rangle_{1}^{*}+\left\langle v_{b}\right\rangle_{1}^{*} e^{j 2 \pi / 3}+\left\langle v_{c}\right\rangle_{1}^{*} e^{-j 2 \pi / 3}\right]
$$

It is clearly seen that the two right-hand side terms define the positive and the negative sequences of input voltage vector.

The latter will appear under unbalanced phase voltages only. In terms of synchronous $d q$ frame defined by (14), the voltage vector (21) can be derived as:

$$
v_{d}+j v_{q}=\vec{v} e^{-j \omega t}=\frac{2}{3}\left[\left\langle v_{a}\right\rangle_{1}+\left\langle v_{b}\right\rangle_{1} e^{j 2 \pi / 3}+\left\langle v_{c}\right\rangle_{1} e^{-j 2 \pi / 3}\right]+\frac{2}{3} e^{-j 2 \omega t}\left[\left\langle v_{a}\right\rangle_{1}^{*}+\left\langle v_{b}\right\rangle_{1}^{*} e^{j 2 \pi / 3}+\left\langle v_{c}\right\rangle_{1}^{*} e^{-j 2 \pi / 3}\right]
$$

Re-arranging the right-side terms results in: 


$$
v_{d}+j v_{q}=\left(V_{d 0}+j V_{q 0}\right)+\left(V_{d 2}+j V_{q 2}\right) e^{-j 2 \omega t}
$$

where variables $V_{d 0}, V_{q 0}, V_{d 2}$ and $V_{q 2}$ can be calculated as:

$$
\begin{aligned}
& V_{d 0}=\frac{2}{3} \operatorname{Re}\left[\left\langle v_{a}\right\rangle_{1}+\left\langle v_{b}\right\rangle_{1} e^{j 2 \pi / 3}+\left\langle v_{c}\right\rangle_{1} e^{-j 2 \pi / 3}\right] \\
& V_{q 0}=\frac{2}{3} \operatorname{Im}\left[\left\langle v_{a}\right\rangle_{1}+\left\langle v_{b}\right\rangle_{1} e^{j 2 \pi / 3}+\left\langle v_{c}\right\rangle_{1} e^{-j 2 \pi / 3}\right] \\
& V_{d 2}=\frac{2}{3} \operatorname{Re}\left[\left\langle v_{a}\right\rangle_{1}^{*}+\left\langle v_{b}\right\rangle_{1}^{*} e^{j 2 \pi / 3}+\left\langle v_{c}\right\rangle_{1}^{*} e^{-j 2 \pi / 3}\right] \\
& V_{q 2}=\frac{2}{3} \operatorname{Im}\left[\left\langle v_{a}\right\rangle_{1}^{*}+\left\langle v_{b}\right\rangle_{1}^{*} e^{j 2 \pi / 3}+\left\langle v_{c}\right\rangle_{1}^{*} e^{-j 2 \pi / 3}\right]
\end{aligned}
$$

The $d$ - and $q$ - axes components of the input voltage vector $v_{d}$ and $v_{q}$ can be derived from (23) by separation of real and imaginary parts:

$$
\begin{aligned}
& v_{d}=V_{d 0}+V_{d 2} \cos 2 \omega t+V_{q 2} \sin 2 \omega t \\
& v_{q}=V_{q 0}+V_{q 2} \cos 2 \omega t-V_{d 2} \sin 2 \omega t
\end{aligned}
$$

The following important conclusions should be made analysing the result given by (25):

- under balanced conditions the voltage $d q$-components become dc-like: $v_{d}=V_{d 0}$ and $v_{q}=V_{q 0}$;

- if the supply voltage is unbalanced, the $d q$ frame components of the voltage vector will also include the second harmonics;

- the DP set $K$, in order to represent the supply voltage in $d q$ frame under both balanced and unbalanced conditions, should include zero and $2^{\text {nd }}$ harmonics, i.e.

$$
K=\{0,2\}
$$

The mapping of $v_{d}$ and $v_{q}$ into DPs is established in Table I. The current $i_{d}$ and $i_{q}$ can be derived in the same manner.

TABLE I. DYNAMIC PHASORS FOR DB INPUT VOLTAGE AND CURRENT IN SYNCHRONOUSLY ROTATING FRAME

\begin{tabular}{ccc}
\hline \hline \multirow{2}{*}{ Variable } & \multicolumn{2}{c}{ Dynamic phasors } \\
\cline { 2 - 3 } & \multicolumn{1}{c}{$k=0$} & \multicolumn{1}{c}{$k=2$} \\
\hline$v_{d}$ & $\left\langle v_{d}\right\rangle_{0}=V_{d 0}$ & $\left\langle v_{d}\right\rangle_{2}=\left(V_{d 2}-j V_{q 2}\right) / 2$ \\
\hline$v_{q}$ & $\left\langle v_{q}\right\rangle_{0}=V_{q 0}$ & $\left\langle v_{q}\right\rangle_{2}=\left(V_{q 2}+j V_{d 2}\right) / 2$ \\
\hline$i_{d}$ & $\left\langle i_{d}\right\rangle_{0}=I_{d 0}$ & $\left\langle i_{d}\right\rangle_{2}=\left(I_{d 2}-j I_{q 2}\right) / 2$ \\
\hline$i_{q}$ & $\left\langle i_{q}\right\rangle_{0}=I_{q 0}$ & $\left\langle i_{q}\right\rangle_{2}=\left(I_{q 2}+j I_{d 2}\right) / 2$ \\
\hline
\end{tabular}




\section{B. DC-link Voltage in dynamic phasors domain}

In this Section we will transform the DB voltage input-output relation (15a) into the DP domain. The main challenge is due to a non-linear nature of this equation that makes the direct application of DP definition (2) non-analytic. The approach we propose in this paper expands (15a) into a Taylor series with respect to $v_{d}$ and $v_{q}$ followed by the transformation of the truncated series into the DP domain. Considering the DB output voltage as a function of two variables $v_{d}$ and $v_{q}$ as follows:

$$
f_{1}\left(v_{d}, v_{q}\right)=v_{d c}=\frac{3 \sqrt{3}}{\pi} \sqrt{v_{d}^{2}+v_{q}^{2}}
$$

Approximating (27) by the Taylor series requires selection of the operation point. The natural choice is the pair $\left\{v_{d}\right.$, $\left.v_{q}\right\}$ under balanced conditions. Hence, the operating point is defined as $\left\{V_{d 0}, V_{q 0}\right\}$ according to (25). The Taylor expansion of (27):

$$
v_{d c}=k_{0}+\frac{k_{1}}{1 !}\left(v_{d}-V_{d 0}\right)+\frac{k_{2}}{1 !}\left(v_{q}-V_{q 0}\right)+\frac{k_{3}}{2 !}\left(v_{d}-V_{d 0}\right)^{2}+\frac{k_{4}}{2 !}\left(v_{q}-V_{q 0}\right)^{2}+\frac{k_{5}}{2 !}\left(v_{d}-V_{d 0}\right)\left(v_{q}-V_{q 0}\right)+\ldots
$$

where $k_{i}$ are constants depending on the selected operation point. These can be calculated using (24), (27) and are given in Appendix I.

The series (28) can be converted into the frequency domain after suitable truncation. Since $v_{d}$ and $v_{q}$ include harmonics up to the second order in our model, we truncate $3^{\text {rd }}$-order and higher terms in (28). From (28), in the balanced condition $\left(v_{d}=V_{d 0}\right.$ and $\left.v_{q}=V_{q 0}\right)$ the dc-voltage $v_{d c}$ will be constant and equal to $k_{0}$ which is associated with the input voltage positive sequence. Under unbalanced conditions, the negative sequence will appear and disturb the diodes switching function (7). The impact of the negative sequence can be represented as a disturbance to $v_{d c}(27)$ in a form of the second harmonic appearing according to (25) in both $v_{d}$ and $v_{q}$. Applying the DP index set (26) and employing the convolution property (4) to the truncated Taylor series of (28), the DPs of dc-link voltage $v_{d c}$ are derived as follows:

$$
\begin{aligned}
& \left\langle v_{d c}\right\rangle_{0}=k_{0}+k_{3}\left\langle v_{d}\right\rangle_{2}\left\langle v_{d}\right\rangle_{2}^{*}+k_{4}\left\langle v_{q}\right\rangle_{2}\left\langle v_{q}\right\rangle_{2}^{*}+k_{5}\left(\left\langle v_{d}\right\rangle_{2}\left\langle v_{q}\right\rangle_{2}^{*}+\left\langle v_{d}\right\rangle_{2}^{*}\left\langle v_{q}\right\rangle_{2}\right) \\
& \left\langle v_{d c}\right\rangle_{2}=k_{1}\left\langle v_{d}\right\rangle_{2}+k_{2}\left\langle v_{q}\right\rangle_{2}
\end{aligned}
$$

The DPs $\left\langle v_{d}\right\rangle_{0},\left\langle v_{d}\right\rangle_{2},\left\langle v_{q}\right\rangle_{0}$ and $\left\langle v_{q}\right\rangle_{2}$ are given in the Table I in a previous Section. Hence, the DPs for dc-link voltage are fully defined. 


\section{Accounting for the dc-voltage ripple}

The converter output voltage calculated using (29) exhibits only dc-component under balanced conditions and includes the $2^{\text {nd }}$ harmonic under unbalances. For functional-level simulation studies this may be sufficient. However, there is a room for convenient model improvement by inclusion into a model the $6^{\text {th }}$ harmonic on the dc side voltage that exhibits under balanced conditions in the rectifier under consideration [22]. This section demonstrates how this component can be introduced into the DP-based model if required.

Under the balanced operation, the $6^{\text {th }}$ harmonic in the de voltage is due to the $5^{\text {th }}$ and $7^{\text {th }}$ harmonic in the switching function [23] that can be given as:

$$
\begin{aligned}
& S_{a 5,7}=-\frac{2 \sqrt{3}}{5 \pi} \cos (5 \omega t+5 \varphi)+\frac{2 \sqrt{3}}{7 \pi} \cos (7 \omega t+7 \varphi) \\
& S_{b 5,7}=-\frac{2 \sqrt{3}}{5 \pi} \cos \left(5 \omega t+5 \varphi+\frac{2 \pi}{3}\right)+\frac{2 \sqrt{3}}{7 \pi} \cos \left(7 \omega t+7 \varphi-\frac{2 \pi}{3}\right) \\
& S_{c 5,7}=-\frac{2 \sqrt{3}}{5 \pi} \cos \left(5 \omega t+5 \varphi-\frac{2 \pi}{3}\right)+\frac{2 \sqrt{3}}{7 \pi} \cos \left(7 \omega t+7 \varphi+\frac{2 \pi}{3}\right)
\end{aligned}
$$

Combining (30) with (8), (9) and (12), the magnitude of the $6^{\text {th }}$ harmonic in dc voltage $v_{d c}$ and its phase angle $\varphi_{d c 6}$ are derived as follows:

$$
\begin{aligned}
& v_{d c 6}=-\frac{3 \sqrt{3}}{5 \pi} \sqrt{V_{d 0}^{2}+V_{q 0}^{2}}+\frac{3 \sqrt{3}}{7 \pi} \sqrt{V_{d 0}^{2}+V_{q 0}^{2}} \\
& \varphi_{d c 6}=6 \tan ^{-1}\left(V_{q 0} / V_{d 0}\right)
\end{aligned}
$$

In a way similar to (20), one can derive the corresponding DP:

$$
\left\langle v_{d c}\right\rangle_{6}=\frac{1}{2} v_{d c 6} e^{j \varphi_{d c 6}}
$$

This extra DP can be added to the previously derived set (29) to represent the DB dc-link voltage in the DP domain.

The time-domain value of dc-link voltage can be calculated using the definition (1):

$$
v_{d c}(t)=\sum_{k}\left\langle v_{d c}\right\rangle_{k}(t) e^{j k \omega t}=2\left(\Re e\left[\left\langle v_{d c}\right\rangle_{k}\right] \cos k \omega t-\mathfrak{I} m\left[\left\langle v_{d c}\right\rangle_{k}\right] \sin k \omega t\right)
$$

where $k=\{0,2,6\}$. Hence, the dc-link voltage (34) includes the dc component as well as the $2^{\text {nd }}$ and the $6^{\text {th }}$ harmonics. Under balanced conditions the $2^{\text {nd }}$ harmonic is absent. However as the unbalance or line fault occurs, it becomes the dominant harmonic component. 


\section{Rectifier ac currents}

The linear relationship between the magnitude of the current vector $i_{m}$ and the dc-link current $i_{d c}$ was given in (15b) and can be transformed into DP domain as follows:

$$
\left\langle i_{m}\right\rangle_{k}=\frac{2 \sqrt{3}}{\pi}\left\langle i_{d c}\right\rangle_{k}
$$

The ac input currents of the rectifier are dependent on the dc load current which is determined jointly by the dc-link voltage $v_{d c}$ and by the load itself. In the proposed model the dc current is derived from its time-domain value measured at the model output and then converted into the DP. The DP index for $\left\langle i_{d c}\right\rangle_{k}$ for linear loads should be chosen according to $\left\langle v_{d c}\right\rangle_{k}$, i.e. $k=\{0,2,6\}$. However, using the following equation

$$
\left\langle i_{d c}\right\rangle_{0}=i_{d c}
$$

allows us to avoid calculating of $\left\langle i_{d c}\right\rangle_{2}$ and $\left\langle i_{d c}\right\rangle_{6}$ with the DP definition (1) and thus no cumbersome calculation of $\int_{t-T}^{t} x e^{-j k \omega t} d t$ is needed. With (36) all the dc-link current information will be included in $\left\langle i_{d c}\right\rangle_{0}$. The fluctuation of $i_{d c}$ will be reflected to the fundamental DPs $\left\langle i_{a, b, c}\right\rangle_{1}$ through $\left\langle i_{d c}\right\rangle_{0}$ and this will be illustrated later in the paper. From (35), the same DP set $k=0$ will apply to $i_{m}$. The DP for the input current $d$ - and $q$-axis components can be derived using (16) as follows:

$$
\begin{aligned}
& \left\langle i_{d}\right\rangle_{k}=\sum_{i=0}^{k}\left\langle i_{m}\right\rangle_{i}\langle\cos \varphi\rangle_{k-i} \\
& \left\langle i_{q}\right\rangle_{k}=\sum_{i=0}^{k}\left\langle i_{m}\right\rangle_{i}\langle\sin \varphi\rangle_{k-i}
\end{aligned}
$$

The main challenge in calculation of (37) deals with the establishing the DPs for non-linear functions $\sin \varphi \operatorname{and} \cos \varphi$. As in previous Section, the approximation is executed by a Taylor series. The non-linear terms are expressed via $d$ - and $q$ axes voltage components:

$$
\begin{aligned}
& \cos \varphi=f_{2}\left(v_{d}, v_{q}\right)=\frac{v_{d}}{\sqrt{v_{d}^{2}+v_{q}^{2}}} \\
& \sin \varphi=f_{3}\left(v_{d}, v_{q}\right)=\frac{v_{q}}{\sqrt{v_{d}^{2}+v_{q}^{2}}}
\end{aligned}
$$

Selecting the operation point $\left\{V_{d 0}, V_{q 0}\right\}$, using the same technique as that dealing $v_{d c}$ in (27) derives,

$$
\langle\cos \varphi\rangle_{0}=h_{0}+h_{3}\left\langle v_{d}\right\rangle_{2}\left\langle v_{d}\right\rangle_{2}^{*}+h_{4}\left\langle v_{q}\right\rangle_{2}\left\langle v_{q}\right\rangle_{2}^{*}+h_{5}\left(\left\langle v_{d}\right\rangle_{2}\left\langle v_{q}\right\rangle_{2}^{*}+\left\langle v_{d}\right\rangle_{2}^{*}\left\langle v_{q}\right\rangle_{2}\right)
$$




$$
\begin{aligned}
& \langle\cos \varphi\rangle_{2}=h_{1}\left\langle v_{d}\right\rangle_{2}+h_{2}\left\langle v_{q}\right\rangle_{2} \\
& \langle\sin \varphi\rangle_{0}=g_{0}+g_{3}\left\langle v_{d}\right\rangle_{2}\left\langle v_{d}\right\rangle_{2}^{*}+g_{4}\left\langle v_{q}\right\rangle_{2}\left\langle v_{q}\right\rangle_{2}^{*}+g_{5}\left(\left\langle v_{d}\right\rangle_{2}\left\langle v_{q}\right\rangle_{2}^{*}+\left\langle v_{d}\right\rangle_{2}^{*}\left\langle v_{q}\right\rangle_{2}\right) \\
& \langle\sin \varphi\rangle_{2}=g_{1}\left\langle v_{d}\right\rangle_{2}+g_{2}\left\langle v_{q}\right\rangle_{2}
\end{aligned}
$$

where $h_{i}$ and $g_{i}$ are constant coefficients that depends on a selected operation point and given in Appendix I. Hence, the DPs for the non-linear functions (38) are derived. Then, the DPs for DB input current $d$ - and $q$-components can be easily derived using (37). Finally, if the modelling task instead of $d$ - and $q$ - current components requires the current in a form of three-phase ac variables, then the DPs (37) have to be transformed into $a b c$ frame as described in the following Section.

\section{E. DQO to ABC transformation in dynamic phasors domain}

Applying the convolution property to the $a b c / d q$ transform given by (13) and (14) yields:

$$
\left[\begin{array}{l}
\left\langle i_{a}\right\rangle_{1} \\
\left\langle i_{b}\right\rangle_{1} \\
\left\langle i_{c}\right\rangle_{1}
\end{array}\right]=\left\langle\mathbf{T}^{-1}\right\rangle_{1}\left[\begin{array}{l}
\left\langle i_{d}\right\rangle_{0} \\
\left\langle i_{q}\right\rangle_{0}
\end{array}\right]+\left\langle\mathbf{T}^{-1}\right\rangle_{1}^{*}\left[\begin{array}{l}
\left\langle i_{d}\right\rangle_{2} \\
\left\langle i_{q}\right\rangle_{2}
\end{array}\right]
$$

where, $\mathbf{T}^{-1}$ is the generalized inverse matrix $\mathbf{T}$ :

$$
\mathbf{T}^{-1}=\left[\begin{array}{ll}
\cos \omega t, & -\sin \omega t \\
\cos (\omega t-2 \pi / 3), & -\sin (\omega t-2 \pi / 3) \\
\cos (\omega t+2 \pi / 3), & -\sin (\omega t+2 \pi / 3)
\end{array}\right]
$$

Calculation of $\left\langle\mathbf{T}^{-1}\right\rangle_{1}$ requires the DPs for $\cos \omega t$ and $\sin \omega t$. As $\omega$ is constant, the DP index for these will include the only component $k=1$, hence employing (2) one can easily derive:

$$
\left\langle\mathbf{T}^{-1}\right\rangle_{1}=\left[\begin{array}{cc}
0.5 & 0.5 j \\
-1 / 4-j \sqrt{3} / 4 & -j 1 / 4+\sqrt{3} / 4 \\
-1 / 4+j \sqrt{3} / 4 & -j 1 / 4-\sqrt{3} / 4
\end{array}\right]
$$

Using (43), the DP of input currents in $a b c$ frame can be derived easily. Furthermore, applying definition (1), the corresponding time-domain current values can be calculated as well.

\section{F. Model assembling}

The equations derived in the sections above can be combined together in order to build the DP-domain model of the uncontrolled bridge rectifier as it is shown in in Fig. 2. 


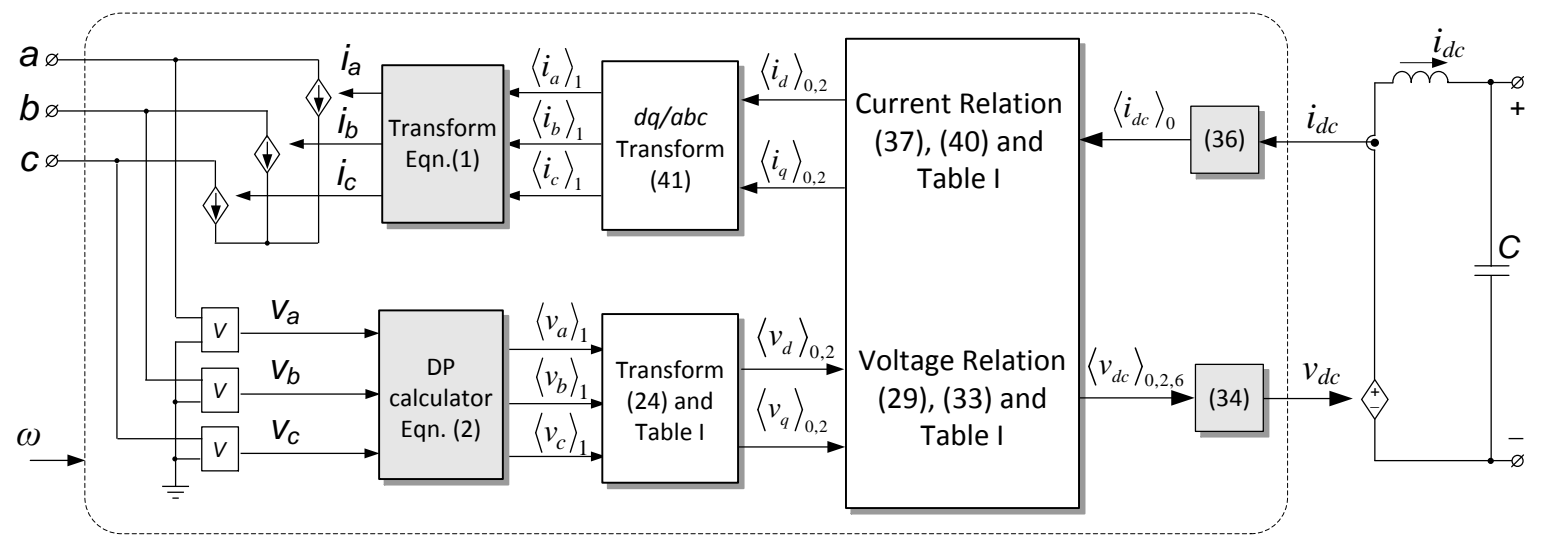

Fig. 2 DP model of the DB rectifier

The current flow shown in Fig.2 illustrates the mapping of $i_{d c}$ into the AC currents $\left\langle i_{a, b, c}\right\rangle_{l}$. With (36), all the information in $i_{d c}$ is reserved in the DP $\left\langle i_{d c}\right\rangle_{0}$. This makes $\left\langle i_{a, b, c}\right\rangle_{1}$ a function of the time-varying current $i_{d c}$ and allows the harmonic characteristics in the ac currents to be represented by the fundamental DPs $\left\langle i_{a, b, c}\right\rangle_{1}$. The DP model shown in Fig. 2 can be used in EPS simulations with no need for the user to understand DP theory. In case studies when the DB rectifier is fed through an inductive line, a small capacitor should be added at input terminals to avoid model state redundancy and related numerical problems. For the user who wishes to build the entire EPS model in the DP domain, the model does not need the interface blocks (coloured in grey in Fig.2) since the DP variables are already available and the DB model can be directly interfaced to the other EPS model blocks.

\section{MODEL ERror ANALYSIS}

In the diode-bridge DP-domain model derived in previous Sections we employed linearization of (27) and (38) around the operation point determined by the voltage positive sequence; the negative sequence was treated as a disturbance. With the increase of the negative sequence this consideration may result in some modelling error that is a subject of analysis in the current paper Section.

Consider the modelling error $\varepsilon$ defined as:

$$
\varepsilon=\frac{\int_{T} v_{d c_{-} B M} d t-\int_{T} v_{d c_{-} D P M} d t}{\int_{T} v_{d c_{-} B M} d t} \times 100 \%
$$

where $v_{d c_{-} B M}$ is the dc-link voltage calculated by the benchmark model (this is a full switching model in three-phase coordinates) and $v_{d c_{-} D P M}$ is the same calculated by the DP model. The analysis has been performed for the discontinuous 
mode case described in the previous Section with the set of phase voltages as:

$$
v_{a}=80 \cos (\omega t), v_{b}=V_{b} \sin \left(\omega t+\phi_{b}\right), v_{c}=80 \cos (\omega t+2 \pi / 3)
$$

Evaluation of the error (44) has been performed by series of simulations varying the magnitude of phase $b$ voltage $V_{b}$ from 0 to $120 \mathrm{~V}$ and its phase $\phi_{b}$ from 0 to $2 \pi$. Thus all balanced and unbalanced conditions are covered. The results of the error analysis are depicted in Fig. 3 as a function of $V_{b}, \phi_{b}$ and the unbalance factor $\lambda$. The latter is defined as the ratio of between the magnitudes of negative-sequence voltage $v_{n}$ and the positive-sequence one $v_{p}$ and written as:

$$
\lambda=v_{n} / v_{p}
$$
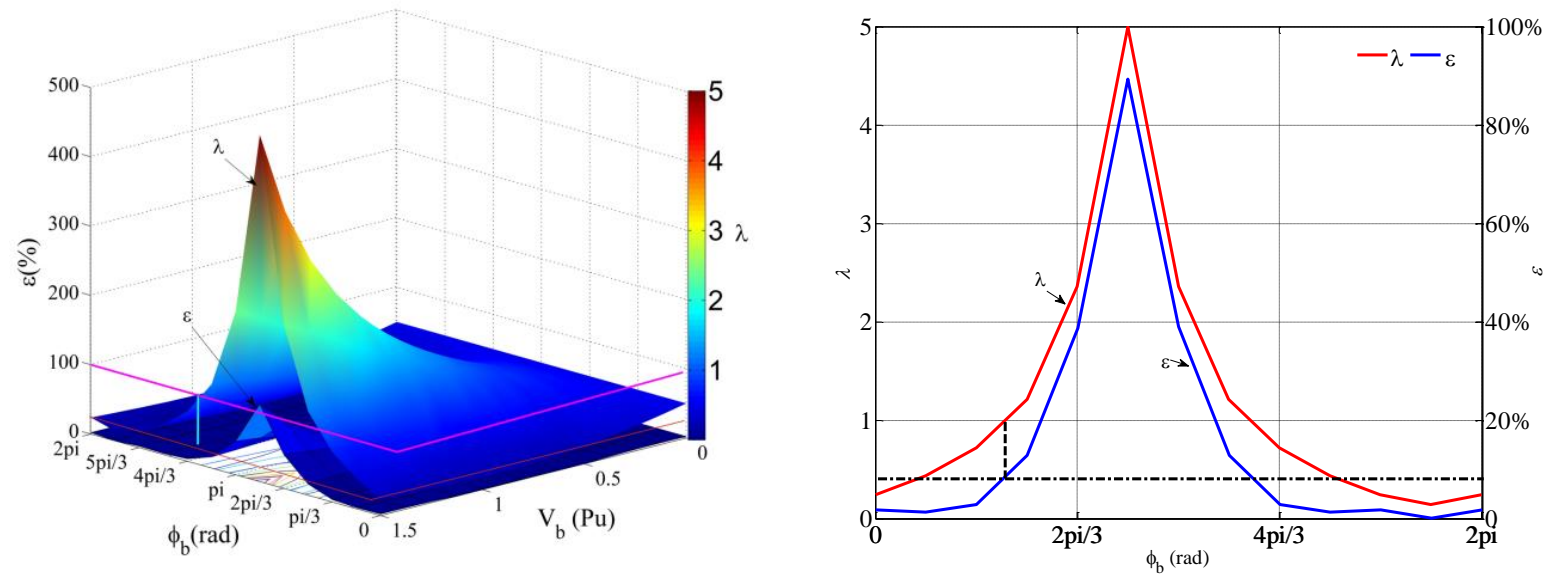

Fig. 3. Analysis of the DP model: (a) - 3D presentation of $\varepsilon=\varepsilon\left(V_{b}, \phi_{b}\right), \lambda=\lambda\left(V_{b}, \phi_{b}\right)$; (b) $-2 \mathrm{D}$ graph showing $\varepsilon=\varepsilon\left(\phi_{b}, \lambda\right)$ with $\mathrm{V}_{\mathrm{b}}=120 \mathrm{~V}$

It is clearly seen that the model error greatly depends on unbalance factor $\lambda$. This is reasonable since with the increase of $\lambda$ the disturbance from the negative sequence becomes more severe and thus the operation point shifts from $\left\{V_{d 0}\right.$, $\left.V_{q 0}\right\}$ as selected for linearization of (27) and (38). The modelling error has a maximum when $\phi_{b}=5 \pi / 6$ and $V_{b}=120 \mathrm{~V}-$ at these values $\lambda$ takes an extreme value. In practice, if the negative sequence becomes dominant, i.e. $\lambda>1$, the operation point should be selected using the negative sequence $\left\{V_{d 2}, V_{q 2}\right\}$ considering the positive sequence as a disturbance. Thus, the model can be conveniently adopted for this case. Hence in the error analysis we only have to consider the cases when $\lambda \in[0,1]($ below the horizontal line $\lambda=1$ in Fig. 3(b)) . For the case of balanced operation $\lambda=0$ the modelling error is less than $2 \%$. The line-to-line fault can be considered as the case when $\lambda=1$ and $V_{b}=120 \mathrm{~V}$; the modelling error for this event is less than $10 \%$ as follows from Fig. 3(b). For the case of phase-to-ground fault $\left(V_{b}=0 \mathrm{~V}\right)$ the modelling error is very small and is always less than $2 \%$.

The modelling accuracy analysis confirms that the developed DP model is well suitable for the functional modelling of uncontrolled diode-bridge rectifier. The accuracy of the DP model will be experimentally validated and discussed in the 
following section.

\section{MODEL EXPERIMENTAL VALIDATION}

A test rig shown in Fig.4 with parameters given in Appendix III has been employed for the model verification. A programmable source Chroma II has been used to apply balanced and unbalanced voltages to the DB. The model has also been verified in continuous and discontinuous current modes as reported below. In the simulation model, small capacitors $(\mathrm{C}=1 \mathrm{e}-8 \mathrm{~F})$ were added between the diode bridge and the line inductors as suggested in Section IV.F above.

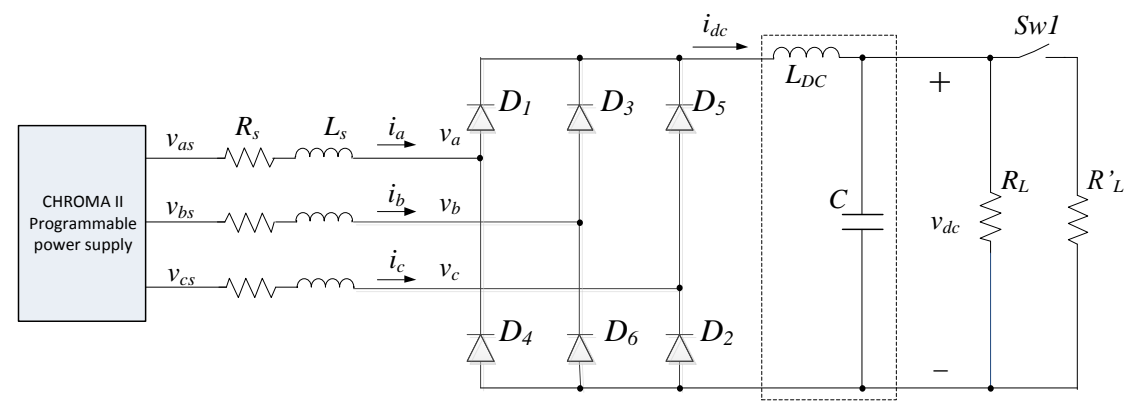

Fig.4. The experimental rig

\section{A. Continuous mode}

In the experiment, the initial value of balanced supply voltage was set to values $v_{a}=v_{b}=v_{c}=40 \mathrm{~V} / 50 \mathrm{~Hz}$ to avoid source protection triggering when imitating the fault. The phase A voltage is set to zero at $t=0.2 \mathrm{~s}$ in order to perform the lineto-ground fault to the system. A continuous current mode is ensured with a heavy load on dc-link side by switching on $S w 1$ and paralleling $R_{L}$ and $R_{L}^{\prime}$ ). The simulation and experiment values of dc-link voltage, input currents and the inductor current are compared in Fig. 5.
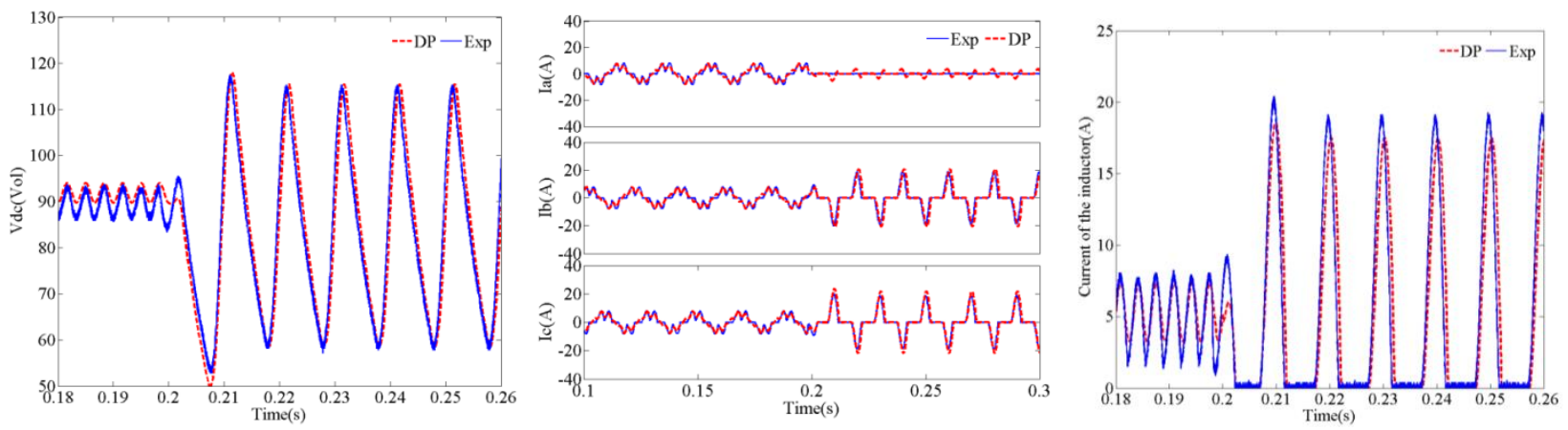

Fig. 5. The dc-link voltage (a), input current (b) and inductor current (c) in continuous mode with phase A loss at $t=0.2 \mathrm{~s}$

As can be seen in Fig. 5(a), under the balanced condition $t<0.2 \mathrm{~s}, v_{\mathrm{dc}}$ has the $6^{\text {th }}$ harmonic component. With the $6^{\text {th }}$ 
harmonic included in the DP model, the results from the experiment and DP model are well matched. After the line-toground fault, the dc-link voltage fluctuates at double frequency. In this case, the $2^{\text {nd }}$ harmonic is included in the DP model and the results from the experiment and simulation are well matched. The DP model and experiment results for $i_{a, b, c}$ are shown in Fig. 5(b) and they are well matched before and after the fault occurs. The DC-link current $i_{d c}$ is shown in Fig. 5(c) and it changes from a continuous current mode (CCM) to a discontinuous mode (DCM) when the fault occurs. It can be seen that under CCM and DCM conditions, the DP model demonstrates good performance in both cases.

\section{B. Discontinuous mode}

In this case the switch $S w 1$ is open hence a large load resistance $R_{L}$ results in a small dc-link current which makes the inductor current discontinuous. The experiment starts with balanced voltages set $v_{a}=v_{b}=v_{c}=80 \mathrm{~V} / 50 \mathrm{~Hz}$ followed the loss of phase A supply $\left(v_{a}=0\right)$ at $t=0.2 \mathrm{~s}$. The simulation and experiment values of dc-link voltage, input currents and the inductor current are compared in Fig. 6.
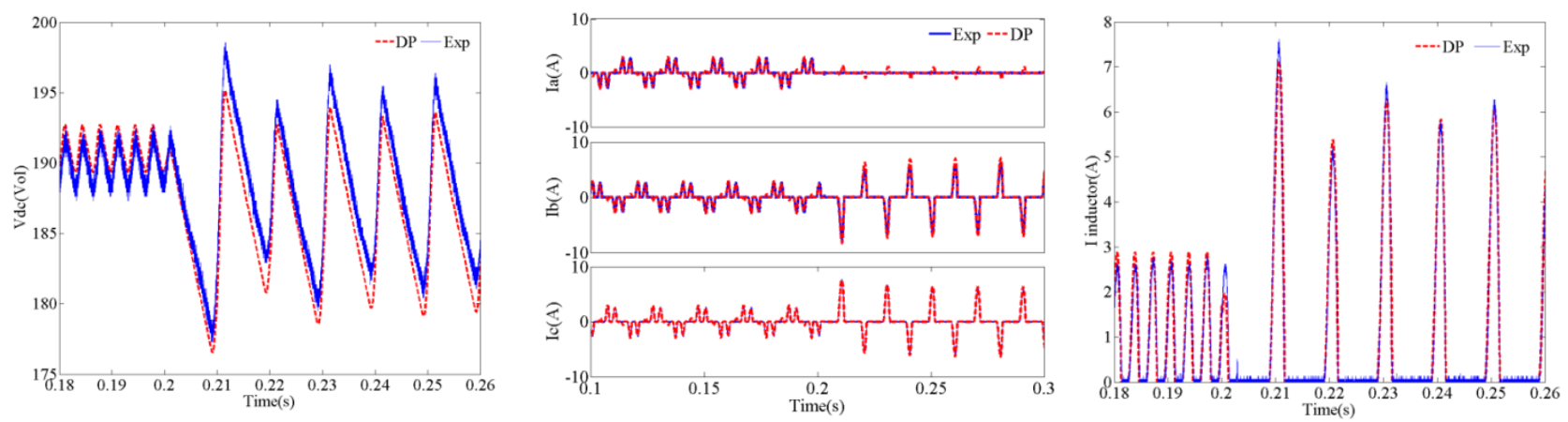

Fig. 6 The dc-link voltage (a), input current (b) and inductor current (c) in discontinuous mode with phase A loss at $t=0.2 \mathrm{~s}$

As can be seen in Fig. 6(a), $v_{d c}$ has a $6^{\text {th }}$ harmonic under the balanced condition and a $2^{\text {nd }}$ harmonic under the line fault condition. In both cases, the results from the DP model are well matched with experiment. The ac side currents from the experiment and the DP model, shown in Fig. 6(b), are well matched before and after the fault occur. The dc-link current $i_{d c}$, shown in Fig. 6(c), indicates that the rectifier works under the DCM under both normal and faulty conditions. The results from the experiment and DP model are well matched in both cases.

Following the results in this section, the proposed model successfully verified under balanced and unbalanced operation, in both continuous and discontinuous current mode. 


\section{COMPUTATION TIME STUDIES}

Since the accuracy of the DP model has been validated, this section will focus on the assessment of the computational performance of the developed DP model. For this purpose, different modelling techniques have been applied to an example EPS given in Fig.7 and the CPU time taken for simulation run has been compared. The simulated scenario includes balanced and unbalanced operation, as well as continuous and discontinuous DB modes as detailed below. The system parameters are given in the Appendix III.

a)

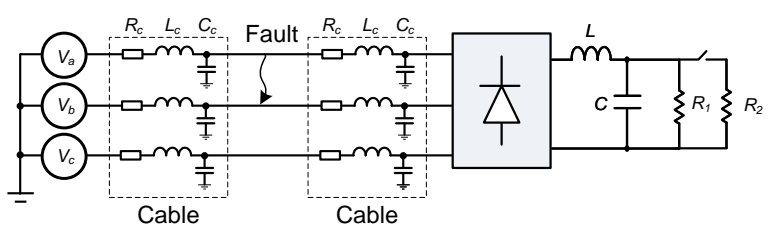

c)

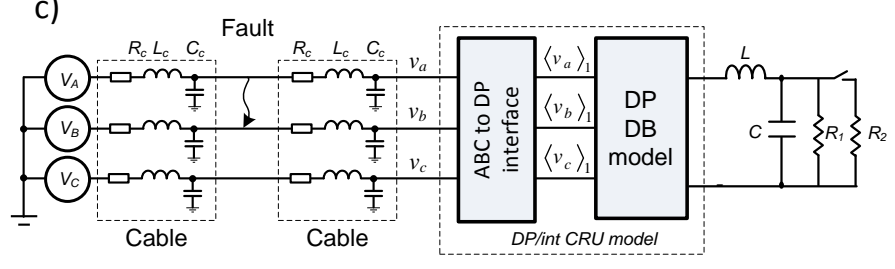

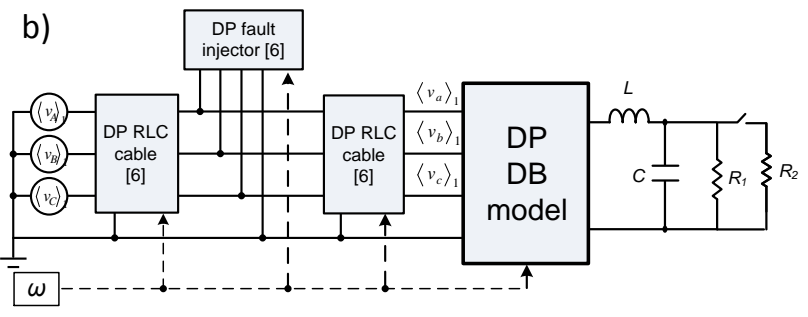

d)

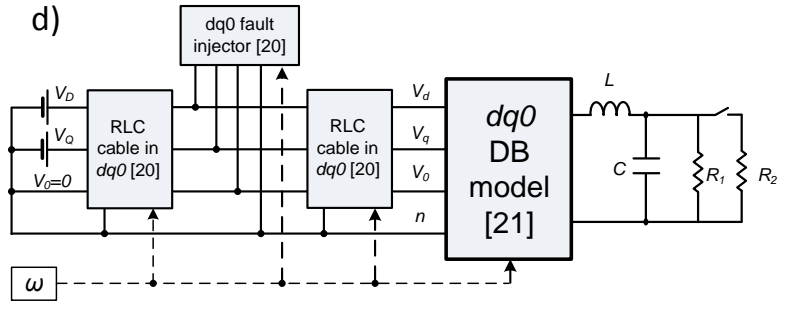

Fig. 7 EPS models for comparison: (a) $a b c$ model; (b) $D P$ model; (c) $D P / i n t$ model; (d) $d q 0$ model.

The following EPS models have been developed and compared:

- A three-phase EPS with a switching DB rectifier model (ideal switches) illustrated by Fig.7(a) and referred to as an $a b c$ model;

- An EPS with all the elements are DP models as shown in Fig.7(b). There are no three-phase time-domain variables in this model and it is referred to as $D P$ model;

- An EPS in which only the DB is modelled using DPs with three-phase interfaces as shown in Fig.7(c). The EPS is seen by the user as a three-phase system and the DP formulations are not visible. This is termed the DP/int model;

- An EPS with all the elements in $d q 0$ frame [24, 25] as shown in Fig.7(d).

\section{A. Models comparison under balanced operation}

The simulation scenario in this case assumes the DB operation in discontinuous model followed by impact of dc load making the dc-link current continuous at $t=0.1 \mathrm{~s}$. The dc-link voltage and current for all the models are shown in Fig.8(a). As one can see, the $D P$ and $D P /$ int models match very well to $a b c$ model in both discontinuous and continuous 
mode. In contrast, the $d q 0$ model delivers correct average $v_{d c}$ and $i_{d c}$ values of $v_{d c}$ only in continuous mode, however in discontinuous mode it demonstrates a significant discrepancy compare to models. This is because in discontinuous mode when $i_{d c}$ becomes zero the capacitor discharges to the load impacting the average value of $v_{d c}$, however the $d q 0$ model does not include the effect of additional charging received due to the $6^{\text {th }}$ harmonic.

The CPU time taken by different models is compared in Table II. The DP and DP/int models in balanced operation are more than 20 times faster than $a b c$ model, however the $d q 0$ model is much faster. The cumulative CPU time during the simulation is shown in Fig.8(b). Note that when the continuous mode occurs the $a b c$ model slows down dramatically but $D P$ and DP/int models maintain the simulation speed. This is because in discontinuous operation there are no commutating periods in DB and only two diodes conduct at any instant, however in continuous mode there are commutation periods requiring from the solver reduction of integration time-steps thus slowing down the simulation.

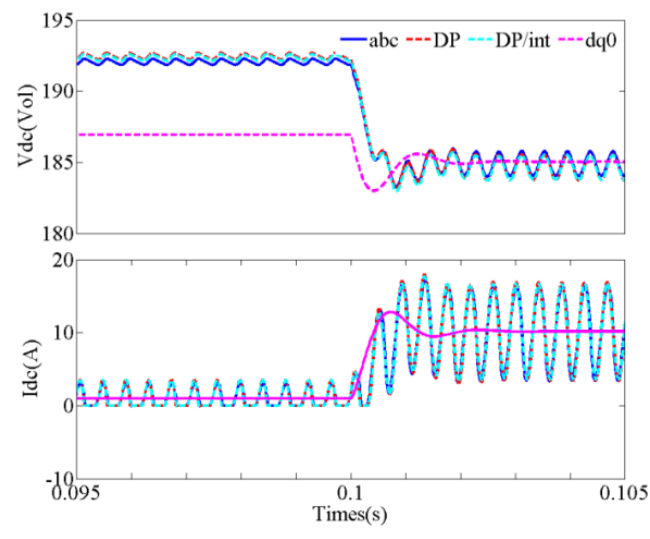

(a)

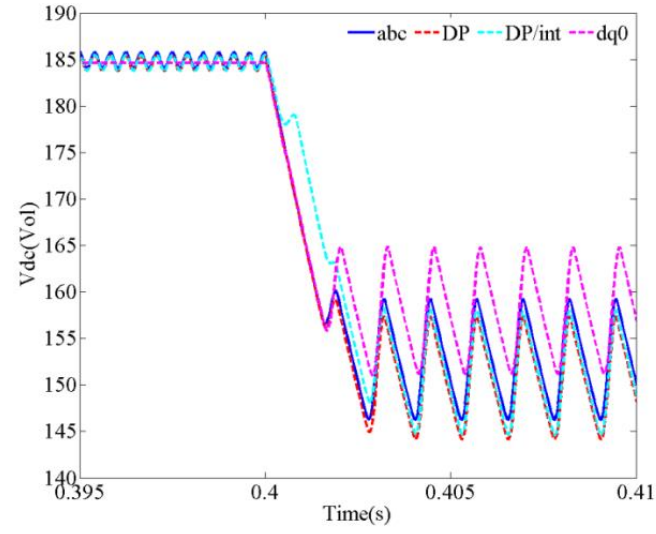

(c)

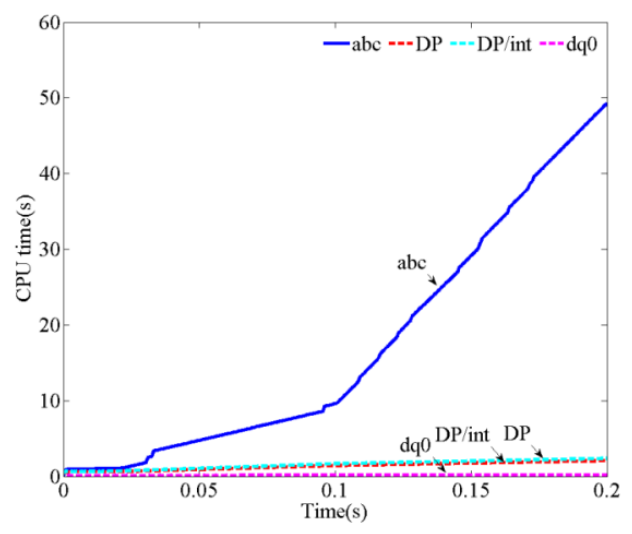

(b)

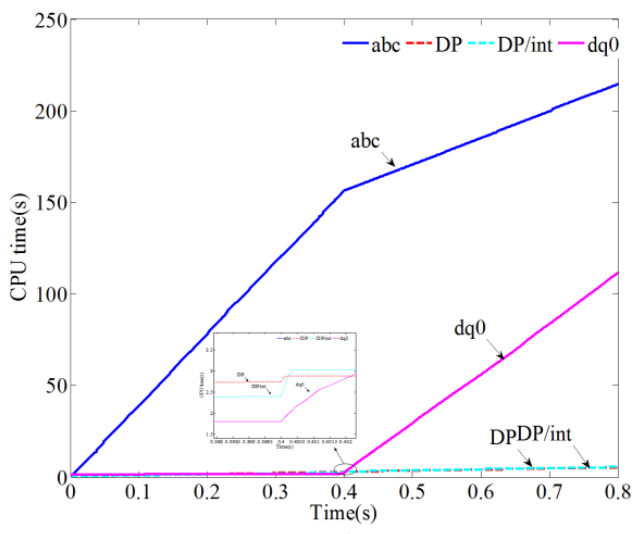

(d)

Fig. 8 Simulation result comparison between different models. Balanced conditions (a) dc-link voltage and current; (b) computation time; Unbalanced conditions (c) dc-link voltage; (d) computation time 


\begin{tabular}{lcccc}
\hline \hline Model: & $a b c$ & $d q 0$ & $D P$ & DP/int \\
\hline CPU time taken: & 49.17 & 0.206 & 2.079 & 2.369 \\
Performance index: & $\mathbf{1}$ & $\mathbf{2 3 8}$ & $\mathbf{2 4}$ & $\mathbf{2 1}$ \\
\hline \hline
\end{tabular}

Summarizing, in balanced conditions the $D P$ and $D P / i n t$ models are slower than $d q 0$ model due to complexity and inclusion of higher harmonics, however the $d q 0$ model is much less accurate especially in discontinuous mode.

\section{B. Line-to-line fault}

The unbalanced regime is simulated as a line-to-line fault (phases A and B are shortened via the fault resistor $R_{\text {faul }}=1 \mathrm{e}-4 \Omega$ ) at $t=0.4 \mathrm{~s}$. The switch $S w 1$ is "on" to ensure the continuous mode prior the fault. The dc-link voltage is shown in Fig.8(c). As one can conclude, the $D P$ and DP/int models match well to $a b c$ model under both balanced and unbalanced regimes. The $d q 0$ model in the balanced condition $(t<0.4 \mathrm{~s})$ reflects the voltage average value.

The CPU time taken by different models is compared Table III. As one can conclude, the efficiency of the $d q 0$ model is lost in unbalanced operation. This is clearly seen from the cumulative CPU time graph in Fig.8(d): the slope corresponding to $d q 0$ model is the largest when $t>0.4 \mathrm{~s}$. In contrast, the $D P$-based models maintain their simulation speed and for the entire scenario they are 40 times faster of compare to the $a b c$ model and 20 times faster than the $d q 0$ model.

Table III. CPU time taken for unbalanced scenario simulation

\begin{tabular}{lcccc}
\hline \hline Model: & $a b c$ & $d q 0$ & $D P$ & DP/int \\
\hline CPU time taken: & 214.4 & 111.4 & 5.405 & 5.448 \\
Performance index: & $\mathbf{1}$ & $\mathbf{2}$ & $\mathbf{4 0}$ & $\mathbf{4 0}$ \\
\hline \hline
\end{tabular}

Hence, the results above have confirmed a very good efficiency of the developed DB model compare to other modelling techniques, in particular - for simulation studies of EPS in unbalanced/line fault conditions.

\section{CONCLUSION}

This paper extends the DP concept into modelling a three-phase diode rectifier. Considering the fact that the $6^{\text {th }}$ harmonic is the dominant harmonic on the DC-link side under balanced conditions and the $2^{\text {nd }}$ harmonic for unbalanced conditions, the developed DP model achieved higher accuracy by embracing the $2^{\text {nd }}$ and $6^{\text {th }}$ harmonics in the DC-link variables. Compare to the traditional average model ( $d q 0$ model), the developed DP-based model is more accurate in the balanced condition and much more time-efficient in the unbalanced condition. The accuracy of developed more is validated using simulation and experiment. In addition, our analysis shows that the maximum error is less than $10 \%$ at the most severe 
unbalanced conditions. The model can be conveniently interfaced with other standard three-phase time-domain model and thus can be widely used in the accelerated electric power system simulation studies.

APPENDIX I. TAYLOR SERIES COEFFICIENTS IN (28)

$k_{0}=f_{1}\left(V_{d 0}, V_{q 0}\right) ; k_{1}=\frac{\partial f_{1}\left(V_{d 0}, V_{q 0}\right)}{\partial v_{d}} ; k_{2}=\frac{\partial f_{1}\left(V_{d 0}, V_{q 0}\right)}{\partial v_{q}} ; k_{3}=\frac{\partial^{2} f_{1}\left(V_{d 0}, V_{q 0}\right)}{\partial v_{d}^{2}} ; k_{4}=\frac{\partial^{2} f_{1}\left(V_{d 0}, V_{q 0}\right)}{\partial v_{q}^{2}} ; k_{5}=2 \frac{\partial^{2} f_{1}\left(V_{d 0}, V_{q 0}\right)}{\partial v_{d} \partial v_{q}}$

\section{APPENDIX II. TAYLOR SERIES COEFFICIENTS IN (39)}

$$
\begin{aligned}
& h_{0}=f_{2}\left(V_{d 0}, V_{q 0}\right) ; h_{1}=\frac{\partial f_{2}\left(V_{d 0}, V_{q 0}\right)}{\partial v_{d}} ; h_{2}=\frac{\partial f_{2}\left(V_{d 0}, V_{q 0}\right)}{\partial v_{q}} ; h_{3}=\frac{\partial^{2} f_{2}\left(V_{d 0}, V_{q 0}\right)}{\partial v_{d}^{2}} ; h_{4}=\frac{\partial^{2} f_{2}\left(V_{d 0}, V_{q 0}\right)}{\partial v_{q}^{2}} ; h_{5}=2 \frac{\partial^{2} f_{2}\left(V_{d 0}, V_{q 0}\right)}{\partial v_{d} \partial v_{q}} ; \\
& g_{0}=f_{3}\left(V_{d 0}, V_{q 0}\right) ; g_{1}=\frac{\partial f_{3}\left(V_{d 0}, V_{q 0}\right)}{\partial v_{d}} ; g_{2}=\frac{\partial f_{3}\left(V_{d 0}, V_{q 0}\right)}{\partial v_{q}} ; g_{3}=\frac{\partial^{2} f_{3}\left(V_{d 0}, V_{q 0}\right)}{\partial v_{d}^{2}} ; g_{4}=\frac{\partial^{2} f_{3}\left(V_{d 0}, V_{q 0}\right)}{\partial v_{q}^{2}} ; g_{5}=2 \frac{\partial^{2} f_{3}\left(V_{d 0}, V_{q 0}\right)}{\partial v_{d} \partial v_{q}}
\end{aligned}
$$

\section{APPENDIX III. EXPERIMENTAL RIG PARAMETERS}

Power source: Chroma, model 61511. DB rectifier: IRKD101-14. AC supply frequency - 50Hz. Input impedance (ac cable and chokes): $L_{S}=1 \mathrm{mH}, R_{S}=0.1 \Omega$. DC-link: $C=2400 \mu \mathrm{F}, L_{d c}=120 \mu \mathrm{H}$. Loads: $R_{L}=200 \Omega, R_{L}^{\prime}=19 \Omega$.

\section{APPENDIX IV. EPS IN FIG.8 PARAMETERS}

Cable: $R_{c}=0.1 \Omega, L_{c}=2 \mu \mathrm{H}, C_{c}=20 \mathrm{pF} ; \mathrm{DC}-$ link: $L=120 \mu \mathrm{H}, C=500 \mu \mathrm{F}, R_{L}=200 \Omega, R^{\prime}=19 \Omega ;$ AC grid frequency $-400 \mathrm{~Hz}$.

\section{ACKNOWLEDGMENT}

This research was conducted in the frame of CleanSky JTI Project, a FP7 European Integrated Project http://www.cleansky.eu. 


\section{REFERENCES}

[1] Moir, I., Seabridge, A : 'Aircraft Systems: mechanical, electrical, and avionics subsystems integration', (John Wiley \& Sons, 3 rd edn., 2008).

[2] Mohan, N., Robbins, W. P., Undeland, T. M., et al.: 'Simulation of power electronic and motion control systems-an overview', Proceedings of the IEEE, 1994, 82, pp. 1287-1302.

[3] Bozhko, S. V., Wu, T., Hill, C. I. et al.: 'Accelerated simulation of complex aircraft electrical power system under normal and faulty operational scenarios', Conf. IECON, 2010, pp. 333-338.

[4] Chiniforoosh, S., Jatskevich, J. Yazdani, A., et al.: 'Definitions and Applications of Dynamic Average Models for Analysis of Power Systems', IEEE Power Delivery, 2010, 25, pp. 2655-2669.

[5] Sanders, S. R., Noworolski, J. M., Liu, X. Z. et al.: 'Generalized averaging method for power conversion circuits', IEEE Power Electronics, 1991, 6, pp. 251-259.

[6] Yang. T, Bozhko, S., Asher, G.: 'Assessment of dynamic phasors modelling technique for accelerated electric power system simulations', Conf. EPE, 2011, pp. 1-9.

[7] Demiray, T., Andersson, G., Busarello,L.: 'Evaluation study for the simulation of power system transients using dynamic phasor models', Conf. Transmission and Distribution Conference and Exposition: Latin America, 2008, pp. 1-6.

[8] Stankovic A. M. , Aydin, T.: 'Analysis of asymmetrical faults in power systems using dynamic phasors', IEEE Power Systems, 2000,15, pp. 1062-1068.

[9] Demiray, T.: '10th Simulation of Power system Dynamics using Dynamic Phasor Models', Conf. Symposium of specialists in electric operational and expansion planning, Florianopolis, 2006.

[10] Stankovic, A. M., Sanders, S. R., Aydin, T.: 'Dynamic phasors in modeling and analysis of unbalanced polyphase AC machines', IEEE Energy Conversion,2002, vol. 17, pp. 107-113.

[11] Demiray, T., Milano, F., Andersson, G.: 'Dynamic Phasor Modeling of the Doubly-fed Induction Generator under Unbalanced Conditions', Conf. Power Tech, 2007, pp. 1049-1054.

[12] Stankovic, A. M., Mattavelli, P., Caliskan, V., et al.: 'Modeling and analysis of FACTS devices with dynamic phasors', Conf. Power Engineering Society Winter Meeting, 2000, 2, pp. 1440-1446.

[13] Mattavelli P., Stankovic, A. M.: 'Dynamical phasors in modeling and control of active filters', Proc. Int. Conf. Circuits and Systems ISCAS, 1999, pp. 278-282 vol.5.

[14] Hannan, M. A., Mohamed, A., Hussain, A.: 'Modeling and power quality analysis of STATCOM using phasor dynamics', Int. Conf. Sustainable Energy Technologies, 2008, pp. 1013-1018.

[15] Qingru, Q., Shousun, C., Ni, V., et al.: 'Application of the dynamic phasors in modeling and simulation of HVDC', Int. Conf Advances in Power System Control, Operation and Management, 2003, pp. 185-190.

[16] Zhu, H., Cai, Z., Liu, H., et al.: 'Hybrid-model transient stability simulation using dynamic phasors based HVDC system model', Electric Power Systems Research, 2006, 76, pp. 582-591.

[17] Di Gerlando, A., Foglia, G. M., Iacchetti, M. F., et al. : 'Comprehensive steady-state analytical model of a three-phase diode rectifier connected to a constant DC voltage source,' Power Electronics, IET, 2013, 6, pp. 1927-1938. 
[19] Marouchos, C., Darwish, M. K., El-Habrouk, M.: 'New mathematical model for analysing three-phase controlled rectifier using switching functions', Power Electronics, IET, 2010, 3, pp. 95-110.

[20] Cross, A. , Baghramian, A, Forsyth, A.: 'Approximate, average, dynamic models of uncontrolled rectifiers for aircraft applications', Power Electronics, IET, 2009,. 2, pp. 398-409.

[21] Rim, C. T., Hu, D. Y., Cho, G. H.: 'Transformers as equivalent circuits for switches: general proofs and transformation-based analyses', IEEE Industry Applications, 1990, 26, pp. 777-785.

[22] Mohan, N., Undeland, T. M., Robbins, W. P.,: 'Power Electronics: Converters, Applications and Design', (John wiley\&Sons, INC, 2003).

[23] Marouchos, C. C., 'The Switching Function Analysis of Power Electronic Circuits'. (The Institution of Engineering and Technology, 2008).

[24] Wu, T., Bozhko, S., Asher, G., et al.: 'Fast Reduced Functional Models of Electromechanical Actuators for More-Electric Aircraft Power System Study', SAE International, 2008.

[25] Wu, T., Bozhko, S. V., Asher, G. M., et al.: 'A fast dynamic phasor model of autotransformer rectifier unit for more electric aircraft', Int. Conf Industrial Electronics, 2009, pp. 2531-2536 